VOL. 11 (2007), 45-49

\title{
On Kirk's Fixed Point Main Theorem for Asymptotic Contractions
}

\author{
Milan R. TAsković
}

\begin{abstract}
We prove that main result of asymptotic contractions by K ir k [J. Math. Anal. Appl. 277 (2003), 645-650, Theorem 2.1, p. 647] has been for the first time proved 17 years ago in Tasković [Fundamental elements of the fixed point theory, ZUNS-1986, Theorem 4, p. 170]. But, the author (and next other authors) this historical fact is to neglect and to ignore.
\end{abstract}

\section{INTRODUCTION}

In recent years a great number of papers have appeared presenting a various generalizations of the well known Banach-Picard contraction principle (via linear and nonlinear conditions). The following result is a statement with nonlinear conditions given in 2003 by W.A. Kirk.

Theorem 1 (Kirk [2]). Let $(X, \rho)$ be a complete metric space, $T: X \rightarrow X$ continuous function, and $\left\{\varphi_{n}\right\}_{n \in \mathbb{N}}$ sequence of continous functions such that $\varphi_{n}: \mathbb{R}_{+}^{0} \rightarrow \mathbb{R}_{+}^{0}:=[0,+\infty)$ and

$$
\rho\left[T^{n}(x), T^{n}(y)\right] \leq \varphi_{n}(\rho[x, y]) \text { for all } x, y \in X,
$$

and $n \in \mathbb{N}$. Assume also that there exists function $\varphi: \mathbb{R}_{+}^{0} \rightarrow \mathbb{R}_{+}^{0}$ such that for any $r>0, \varphi(r)<r, \varphi(0)=0$ and $\varphi_{n} \rightarrow \varphi(n \rightarrow \infty)$ uniformly of the range of $\rho$. If there exists $x \in X$ such that orbit of $T$ at $x$ is bounded, then $T$ has a unique fixed point $\xi \in X$ and all sequences of Picard iterates defined via $T$ converges to $\xi$.

\section{MAIN RESUltS AND FACTS}

Let $X$ be a topological space, $T: X \rightarrow X$, and let $A: X \times X \rightarrow \mathbb{R}_{+}^{0}$. In 1986 Tasković [3] investigated the concept of TCS-convergence in a space

1991 Mathematics Subject Classification. Primary: 54E15, 47H10, 05A15. Secondary: 54E35, 54H25.

Key words and phrases. Metric and topological spaces, TCS-convergence, Complete spaces, Contraction, Asymptotic contraction, Nonlinear conditions for fixed points. 
$X$, i.e., a topological space $X:=(X, A)$ satisfies the condition of TCSconvergence iff $x \in X$ and if $A\left(T^{n} x, T^{n+1} x\right) \rightarrow 0(n \rightarrow \infty)$ implies that $\left\{T^{n}(x)\right\}_{n \in \mathbb{N}}$ has a convergent subsequence.

For $x \in X$ the $\operatorname{set} \mathcal{O}(x, \infty):=\left\{x, T x, T^{2} x, \ldots\right\}$ is called the orbit of $x$. A function $f$ mapping $X$ into reals is a $T$-orbitally lower semicontinuous at the point $p$ iff for all sequences $\left\{x_{n}\right\}_{n \in \mathbb{N}}$ such that $x_{n} \rightarrow p(n \rightarrow \infty)$ it follows that $f(p) \leq \liminf _{n \rightarrow \infty} f\left(x_{n}\right)$. A mapping $T: X \rightarrow X$ is said to be orbitally continuous if $\xi, x \in X$ are such that $\xi$ is a cluster point of $\mathcal{O}(x, \infty)$, then $T(\xi)$ is a cluster point of $T(\mathcal{O}(x, \infty))$.

The following results, given in the next two theorems are given in 1986 by M. R. Tasković [3] as a natural extension of characterization statements of asymptotically conditions of fixed point theorem given in 1985 by Tasković [4]. These results are according to topological spaces.

Theorem 2 (Tasković [3]). Let $T$ be a mapping of topological space $X:=$ $(X, A)$ into itself, where $X$ satisfies the condition of TCS-convergence. Suppose that there exist a sequence of nonnegative real functions $\left\{\alpha_{n}(x, y)\right\}_{n \in \mathbb{N}}$ such that $\alpha_{n}(x, y) \rightarrow 0(n \rightarrow \infty)$ and positive integer $m(x, y)$ such that

$$
A\left(T^{n}(x), T^{n}(y)\right) \leq \alpha_{n}(x, y) \text { for all } n \geq m(x, y),
$$

and for all $x, y \in X$, where $A: X \times X \rightarrow \mathbb{R}_{+}^{0}$. If $x \mapsto A(x, T(x))$ is a $T$-orbitally lower semicontinuous function or $T$ is orbitally continuous and $A(a, b)=0$ implies $a=b$, then $T$ has a unique fixed point $\xi \in X$ and $T^{n}(x) \rightarrow \xi(n \rightarrow \infty)$ for each $x \in X$.

Proof. For $y=T(x)$ from (B) we have that $A\left(T^{n} x, T^{n+1} x\right) \leq \alpha_{n}(x, T x)$ for all $n \geq m(x, y)$, and thus we obtain that $A\left(T^{n} x, T^{n+1} x\right) \rightarrow 0(n \rightarrow \infty)$. This implies (from TCS-convergence) that the sequence of iterates $\left\{T^{n}(x)\right\}_{n \in \mathbb{N}}$ has a convergent subsequence $\left\{T^{n(i)}(x)\right\}_{i \in \mathbb{N}}$ with the limit point $\xi \in X$. Since $x \mapsto A(x, T(x))$ is $T$-orbitally lower semicontinuous we get

$$
A(\xi, T(\xi)) \leq \liminf _{i \rightarrow \infty} A\left(T^{n(i)} x, T^{n(i)+1} x\right)=\liminf _{n \rightarrow \infty} A\left(T^{n} x, T^{n+1} x\right)=0
$$

which implies that $A(\xi, T(\xi))=0$, i.e. $\xi=T(\xi)$. On the other hand, if $T$ is orbitally continuous the proof of previous fact is trivially. We complete the proof by showing that $T$ can have at most one fixed point. Indeed, if we suppose that $\xi \neq \eta$ were two fixed points, then from (B) we have

$$
0<A(\xi, \eta)=A\left(T^{n}(\xi), T^{n}(\eta)\right) \leq \alpha_{n}(\xi, \eta) \text { for } n \geq m(\xi, \eta) ;
$$

taking limits as $n \rightarrow \infty$ we obtain a contradiction. The proof is complete.

Note that, from the preceding proof of Theorem 2, we can give the following local form of this statement. 
Theorem 3 (Localization of (B), Tasković [3]). Let $T$ be a mapping of topological space $X:=(X, A)$ into itself, where $X$ satisfies the condition of TCS-convergence. Suppose that there exist a sequence of nonnegative real functions $\left\{\alpha_{n}(x, y)\right\}_{n \in \mathbb{N}}$ such that $\alpha_{n}(x, T x) \rightarrow 0(n \rightarrow \infty)$ and positive integer $m(x)$ such that

$$
A\left(T^{n}(x), T^{n+1}(x)\right) \leq \alpha_{n}(x, T x) \text { for all } n \geq m(x),
$$

and for every $x \in X$, where $A: X \times X \rightarrow \mathbb{R}_{+}^{0}$. If $x \mapsto A(x, T x)$ is a $T$-orbitally lower semicontinuous function or $T$ is orbitally continuous and $A(a, b)=0$ implies $a=b$, then $T$ has at least one fixed point in $X$.

The proof of this statement is an analogous with the preceding proof of Theorem 2. A brief broof of this statement may be found in Tasković [3].

Annotation. The Theorem 1 is a consequence of Theorem 2 (In this sense in next we give the following proof of this essential fact).

Proof. (Application of Theorem 2).

Suppose that all the conditions of Theorem 1 are satisfied. We prove that all conditions of Theorem 2 are satisfied, too. Since $\varphi: \mathbb{R}_{+}^{0} \rightarrow \mathbb{R}_{+}^{0}$ is a continuous function such that $\varphi(t)<t$ for every $t>0$ and $\varphi(0)=0$, from Wong's lemma ([5], Lemma 4, p. 201) it follows that there exists nondecreasing continuous function $\psi: \mathbb{R}_{+}^{0} \rightarrow \mathbb{R}_{+}^{0}$ such that $\varphi(t)<\psi(t)<$ $t$ for every $t>0$ and $\psi(0)=0$. Let us define $A: X \times X \rightarrow \mathbb{R}_{+}^{0}$ by $A(a, b)=\psi(\rho[a, b])$, and define a sequence of functions $\left\{\alpha_{n}(a, b)\right\}_{n \in \mathbb{N}}$ by $\alpha_{n}(a, b)=\rho\left[T^{n}(a), T^{n}(b)\right]$ for any $a, b \in X$. Since $\psi(t)<t$ we get that

$$
A\left(T^{n}(x), T^{n}(y)\right)=\psi\left(\rho\left[T^{n}(x), T^{n}(y)\right]\right)<\rho\left[T^{n}(x), T^{n}(y)\right]=\alpha_{n}(x, y)
$$

this is that the condition (B) is satisfied. Since $\psi(t)=0$ implies $t=0$, from $A(a, b)=\psi(\rho[a, b])=0$ it follows that $\rho[a, b]=0$, i.e., $a=b$. From the proof given by W.A. Kirk [2] it follows that $\rho\left[T^{n}(x), T^{n}(y)\right] \rightarrow 0(n \rightarrow \infty)$ for all $x, y \in X$. Consequently, $\alpha_{n}(x, y) \rightarrow 0(n \rightarrow \infty)$. Since $T$ and $\psi$ are continuous mappings the function $x \mapsto A(x, T x):=\psi(\rho[x, T x])$ is a $T$-orbitally lower semicontinouos. Since $X$ is a complete metric space it satisfies the condition of TCS-convergence. Applying Theorem 2 we obtain that $T$ has a unique fixed point $\xi \in X$ and all sequences of Picard iterates converge to $\xi$. The proof is complete.

Further, applying the Theorem 2 we get an asymptotic version of a statement due to Ivanov [1]. This is the following result which is an extension of Kirk's theorem on asymptotic contractions.

Theorem 4. Let $(X, \rho)$ be a complete metric space, $T: X \rightarrow X$ a continuous function, and $\varphi_{n}: \mathbb{R}_{+}^{0} \rightarrow \mathbb{R}_{+}^{0}$ for $n \in \mathbb{N}$ a sequence such that for all 
$n \in \mathbb{N}$ satisfy

(1) $\rho\left[T^{n}(x), T^{n}(y)\right] \leq$

$\leq \max \left\{\varphi_{n}(\rho[x, y]), \varphi_{n}(\rho[x, T x]), \varphi_{n}(\rho[y, T y]), \varphi_{n}(\rho[x, T y]), \varphi_{n}(\rho[y, T x])\right\}$

for all $x, y \in X$; and assume also that there exists a function $\varphi: \mathbb{R}_{+}^{0} \rightarrow \mathbb{R}_{+}^{0}$ such that for any $t>0, \varphi(t)<t, \varphi(0)=0$ and $\varphi_{n} \rightarrow \varphi(n \rightarrow \infty)$ uniformly of the range of $\rho$. If there exists $x \in X$ such that orbit of $T$ at $x$ is bounded, then $T$ has a unique fixed point $\xi \in X$ and all sequences of Picard iterates defined by $T$ converges to $\xi$.

Proof. (Application of Theorem 2).

Again, since $\varphi: \mathbb{R}_{+}^{0} \rightarrow \mathbb{R}_{+}^{0}$ is a continuous function such that $\varphi(t)<t$ for every $t>0$ and $\varphi(0)=0$, from Wong's lemma ([5] Lemma 4, p. 201) it follows that there exists nondecreasing continuous function $\psi: \mathbb{R}_{+}^{0} \rightarrow \mathbb{R}_{+}^{0}$ such that $\varphi(t)<\psi(t)<t$ for every $t>0$ and $\psi(0)=0$. We define a function $A: X \times X \rightarrow \mathbb{R}_{+}^{0}$ by

$$
A(a, b):=\max \{\psi(\rho[a, b]), \psi(\rho[a, T a]), \psi(\rho[b, T b]), \psi(\rho[a, T b]), \psi(\rho[b, T a])\}
$$

and define a sequence of functions $\left\{\alpha_{n}(x, y)\right\}_{n \in N}$ by

$$
\begin{array}{r}
\alpha_{n}(x, y):=\max \left\{\rho\left[T^{n} x, T^{n} y\right], \rho\left[T^{n} x, T^{n+1} x\right], \rho\left[T^{n} y, T^{n+1} y\right],\right. \\
\left.\rho\left[T^{n} x, T^{n+1} y\right], \rho\left[T^{n} y, T^{n+1} x\right]\right\} .
\end{array}
$$

It is easy to show that $A$ and $\left\{\alpha_{n}(x, y)\right\}_{n \in \mathbb{N}}$ satisfy all the required hypothesis (similarly as in the proof of Kirk's theorem) in Theorem 2. Applying Theorem 2 we get conclusion of Theorem 4. This completes the proof.

\section{REFERENCES}

[1] A.A. Ivanov, Isledovanii po topologii-II, Zapiski naučnih seminarov lomi, Lenjingrad, 66 (1976), 5-102

[2] W.A. Kirk, Fixed points of asymptotic contractions, Journ. Math. Anal. Appl. 277 (2003), 645-650.

[3] M.R. Tasković, Fundamental elements of the fixed point theory, Mat. Biblioteka 50 (Beograd), English sumary: 268-271, Zavod za udžbenike i nastavna sredstva-Beograd, in Serbian, 50 (1986), 274 pages.

[4] M.R. Tasković, A characterization of the class of contraction type mappings, Kobe J. Math., 2(1985), 45-55.

[5] C.S. Wong, Maps of contractive type, Proc. Seminar of Fixed Point Theory and its Appl., Dalhousie University, June 1975, 197-207. 
Milan R. TASKOViĆ

FaCUlty of Mathematics

11000 Belgrade, P.O. Box 550

SERBIA

Home Address:

Milan R. TAsković

NeHRUOVA 236

11070 BELGRADE

SERBIA

E-mail address: andreja@predrag.us 